\title{
The Use of Artificial Neural Networks for the Prediction of Surgical Site Infection Following TKA
}

\author{
Ingwon Yeo, MD ${ }^{1}$ Christian Klemt, $\mathrm{PhD}^{1}$ Matthew Gerald Robinson, MD ${ }^{1}$ John G. Esposito, MD ${ }^{1}$ \\ Akachimere Cosmas Uzosike, MD, $\mathrm{MPH}^{1}$ Young-Min Kwon, MD, $\mathrm{PhD}^{1}$

\footnotetext{
${ }^{1}$ Department of Orthopedic Surgery, Bioengineering Laboratory, Massachusetts General Hospital, Harvard Medical School, Boston, Massachusetts
} \\ J Knee Surg 2023;36:637-643. \\ Address for correspondence Young-Min Kwon, MD, PhD, Department \\ of Orthopedic Surgery, Massachusetts General Hospital, Harvard \\ Medical School, 55 Fruit Street, Boston, MA 02114 \\ (e-mail: ymkwon@mgh.harvard.edu).
}

\author{
Abstract \\ Keywords \\ - total knee \\ arthroplasty \\ - surgical site infection \\ - machine learning \\ - artificial intelligence
}

This is a retrospective study. Surgical site infection (SSI) is associated with adverse postoperative outcomes following total knee arthroplasty (TKA). However, accurately predicting SSI remains a clinical challenge due to the multitude of patient and surgical factors associated with SSI. This study aimed to develop and validate machine learning models for the prediction of SSI following primary TKA. This is a retrospective study for patients who underwent primary TKA. Chart review was performed to identify patients with superficial or deep SSIs, defined in concordance with the criteria of the Musculoskeletal Infection Society. All patients had a minimum follow-up of 2 years (range: 2.1-4.7 years). Five machine learning algorithms were developed to predict this outcome, and model assessment was performed by discrimination, calibration, and decision curve analysis. A total of 10,021 consecutive primary TKA patients was included in this study. At an average follow-up of $2.8 \pm 1.1$ years, SSIs were reported in 404 (4.0\%) TKA patients, including 223 superficial SSIs and 181 deep SSIs. The neural network model achieved the best performance across discrimination (area under the receiver operating characteristic curve $=0.84$ ), calibration, and decision curve analysis. The strongest predictors of the occurrence of SSI following primary TKA, in order, were Charlson comorbidity index, obesity (BMI $\left.>30 \mathrm{~kg} / \mathrm{m}^{2}\right)$, and smoking. The neural network model presented in this study represents an accurate method to predict patient-specific superficial and deep SSIs following primary TKA, which may be employed to assist in clinical decision-making to optimize outcomes in at-risk patients.
In the United States alone, over 680,000 total knee arthroplasty (TKA) procedures are performed each year, with growth projections until 2030 ranging from 84.9 to $147 \%{ }^{1}$ Surgical site infection (SSI) is one of the most common health careassociated infections (HAI) among orthopaedic patients, ${ }^{2}$ estimated to occur in 1 to $3.5 \%$ of total joint arthroplasties (TJAs). ${ }^{3}$ By 2030, the prevalence of TKA patients with SSI will increase due to the significantly increasing demand for primary TKA

received

August 17, 2021

accepted after revision

November 16, 2021

article published online

January 11, 2022 procedures; nonetheless, the rate of SSI following primary TKA is expected to range between 2.2 and $6.8 \%{ }^{4}$ The American College of Surgeons National Surgical Quality Improvement Program divides SSI into superficial and deep SSIs, with deep SSI also commonly being known as periprosthetic joint infection (PJI). While superficial SSI generally requires a less aggressive treatment approach, PJI often necessitates invasive management with intravenous antibiotics and reoperations (c) 2022. Thieme. All rights reserved. Thieme Medical Publishers, Inc., 333 Seventh Avenue, 18th Floor, New York, NY 10001, USA
DOI https://doi.org/ 10.1055/s-0041-1741396. ISSN 1538-8506. 
including TKA component exchanges. ${ }^{5} \mathrm{PJI}$ is the most common indication of revision in TKA patients, ${ }^{6}$ which results in increased length of hospital stay and resource utilization. From a patient's standpoint, superficial and deep SSIs following TKA have been associated with significant morbidity and mortality.

Although the understanding of how patients differ in terms of their susceptibility to SSI prior to TKA remains incomplete, previous studies have identified several risk factors that can be categorized as modifiable and nonmodifiable. ${ }^{7}$ Despite the continuous development of infection prevention modalities such as patient optimization, advances in surgical technique, sterile protocols, and operative procedures, SSI after TKA still poses a substantial burden to patients, surgeons, and the health care system. Therefore, recent studies have developed predictive risk calculators to estimate the probability of SSI preoperatively in an effort to reduce SSI following TKA; however, their study findings demonstrate limited success. ${ }^{8}$ Additionally, these risk calculators provide limited clinical utility due to their cumbersome nature. With the development of computational science and its availability to clinical fields, machine learning (ML) models such as artificial neural networks (ANNs) represent a form of artificial intelligence that is particularly suited for preoperative medical risk stratification and resource allocation. ML models have demonstrated high accuracy in predicting SSI following lumbar spinal fusion. ${ }^{9}$ However, there is a paucity of studies utilizing ML models for the prediction of SSI following TKA. Therefore, this study aimed to develop and validate ML models for the prediction of SSI in patients following primary TKA.

\section{Materials and Methods}

\section{Patient Cohort}

This present study was approved by the Institutional Review Board. A retrospective review of 10,089 primary TKA procedures was performed. All TKA surgeries were performed between 2016 and 2019 at our tertiary academic center. All TKA surgeries were performed by a total of 11 fellowshiptrained arthroplasty surgeons. Patients with simultaneous bilateral surgery, partial joint arthroplasty, and missing perioperative data were excluded from the analysis. All patients had a minimum follow-up of 2 years (range: range: 2.1-4.7 years). A total of 10,021 primary TKA patients remained for the development and validation of ML algorithms to predict SSI following primary TKA. The primary outcome of interest in this study was the prediction of superficial and deep SSIs in patients following primary TKA. SSI was defined in concordance with the criteria of the Musculoskeletal Infection Society. ${ }^{10}$

\section{Clinical Variables}

Using our institution's electronic medical record system for patient chart review, patient and procedural variables associated with the development of SSI following TKA were collected. Collected patient variables included age, gender, body mass index (BMI), ethnicity, insurance status (Medicare, Medicaid, and Private), social status, American Society of Anesthesiologist Physical Status score (ASA score), medical comorbidities, Charlson comorbidity index (CCI), and preoperative medications (-Table 1). Procedural variables included for analysis involved laterality, indication for primary TKA, prior injections/surgeries on the knee, prior ambulatory/inpatient stays, anesthesia type, tranexamic acid usage, component

Table 1 Baseline characteristics of study population

\begin{tabular}{|c|c|}
\hline Characteristic & $\begin{array}{l}\text { Primary TKA patients } \\
(N=10,021)\end{array}$ \\
\hline \multicolumn{2}{|l|}{ Demographics } \\
\hline Age $(y)$ & $74.2 \pm 22.7$ \\
\hline Gender & 3,992 males; 6,029 females \\
\hline BMI $\left(\mathrm{kg} / \mathrm{m}^{2}\right)$ & $32.3 \pm 6.4$ \\
\hline \multirow[t]{2}{*}{ Laterality } & 4,727 left; 5,294 right \\
\hline & ASA $1-616(6.1 \%)$ \\
\hline \multirow[t]{3}{*}{ ASA score (\%) } & ASA 2-6,168 (61.5\%) \\
\hline & ASA 3-3,079 (30.2\%) \\
\hline & ASA 4-226 (2.2\%) \\
\hline $\begin{array}{l}\text { Charlson comorbidity } \\
\text { index }\end{array}$ & $1.9 \pm 1.5$ \\
\hline $\begin{array}{l}\text { Insurance status (Medi- } \\
\text { care; Medicaid; Private) }\end{array}$ & 1,$847 ; 561 ; 7,613$ \\
\hline $\begin{array}{l}\text { Ethnicity (White, African } \\
\text { American, Hispanic, and } \\
\text { Asian) }\end{array}$ & $9,686,163,112,60$ \\
\hline Follow-up time $(y)$ & $2.8 \pm 1.1$ \\
\hline \multicolumn{2}{|l|}{ Comorbidities } \\
\hline Smoking (\%) & $502(5.0 \%)$ \\
\hline Drinking (\%) & $1,413(14.1 \%)$ \\
\hline Drug abuse (\%) & $173(0.1 \%)$ \\
\hline Diabetes mellitus (\%) & $710(7.8 \%)$ \\
\hline Depression (\%) & $594(6.0 \%)$ \\
\hline Renal failure (\%) & $438(4.8 \%)$ \\
\hline Malignant tumor (\%) & $819(8.1 \%)$ \\
\hline Hypertension & $4,648(46.3 \%)$ \\
\hline \multicolumn{2}{|l|}{ Surgical variables } \\
\hline Blood loss (mL) & $109.0 \pm 96.0$ \\
\hline Operation time (min) & $78.9 \pm 32.6$ \\
\hline Spinal anesthesia (\%) & 82.8 \\
\hline $\begin{array}{l}\text { Tranexamic acid usage } \\
\text { (\%) }\end{array}$ & 77.1 \\
\hline Tourniquet use (\%) & 94.1 \\
\hline Transfusion rates (\%) & 3.8 \\
\hline $\begin{array}{l}\text { Cemented component } \\
\text { fixation }(\%)\end{array}$ & 96.2 \\
\hline $\begin{array}{l}\text { Indication for primary } \\
\text { TKA (osteoarthritis) }\end{array}$ & 92.5 \\
\hline
\end{tabular}

Abbreviations: ASA, American Society of Anesthesiologist Physical Status score; BMI, body mass index; TKA, total knee arthroplasty. 
Table 2 Causative pathogens for the development of surgical site infection following primary total knee arthroplasty

\begin{tabular}{|l|l|}
\hline Causative pathogen & $\begin{array}{l}\text { Revision surgery } \\
\text { for SSI (N=181) }\end{array}$ \\
\hline Unfavorable & $10(5.5 \%)$ \\
\hline Methicillin-resistant & $8(4.4 \%)$ \\
\hline Pseudomonas aeruginosa & $10(5.5 \%)$ \\
\hline Anaerobes & $29(16.0 \%)$ \\
\hline Negative culture & $13(7.1 \%)$ \\
\hline Other gram-negative organisms & $20(11.0 \%)$ \\
\hline Mixed growth & $17(9.4 \%)$ \\
\hline Favorable & $15(8.3 \%)$ \\
\hline Streptococcus species & $9(4.9 \%)$ \\
\hline Staphylococcus species & $14(7.7 \%)$ \\
\hline Coagulase-negative Staphylococci & $8(4.4 \%)$ \\
\hline Other gram-positive organisms & $23(12.7 \%)$ \\
\hline Propionibacterium acnes & $5(2.7 \%)$ \\
\hline Staphylococcus aureus & \\
\hline Other & \\
\hline
\end{tabular}

Abbreviation: SSI, surgical site infection.

fixation method (cemented vs. non-cemented), blood loss, transfusion rates, and tourniquet use.

\section{Model Development}

For the SSI classification analysis, we employed five state-ofthe-art supervised ML methods: (1) ANN, (2) stochastic gradient boosting, (3) support vector machines (SVMs), (4) random forest (RF), and (5) elastic-net penalized logistic regression. These ML methods were selected based on prior studies showing the potency of these modeling techniques to accurately predict arthroplasty patient outcomes. The dataset underwent a random division into two groups using an
80:20 stratified split ratio, which resulted in a training dataset (8,016 TKAs) and a testing dataset (2,005 TKAs). Recursive feature elimination was used to select the subset of parameters for final modeling. Five-fold cross-validation was performed five times to develop and assess all candidate models.

The five ML models were assessed using the area under the receiver operating characteristic curve (AUC). An AUC of 1 represents a perfect ML model, while ML models no better than chance have an AUC of $0.5 .^{8}$ ML model calibration was achieved through the use of a calibration plot. Overall model performance was assessed using the Brier score. ${ }^{11}$ Perfect ML models have a Brier score of 0 . Decision curve analysis was performed to measure the expected utility of TKA candidate model predictions if clinical management was to change based on ML model predictions. The interpretability of ML models was performed at both global and local levels.

\section{Statistical Analysis}

All statistical analysis was performed using SPSS (SPSS version 18.0; IBM Corp., Armonk, NY), Matlab (MathWorks Inc., Natick, MA), Python (Python Software Foundation, Wilmington, DE), and Anaconda (Anaconda Inc., Austin, TX).

\section{Results}

A total of 10,021 patients who underwent primary TKA were analyzed. Of those 10,021 patients, 404 (4.0\%) incidences of SSI were observed at an average follow-up of $2.8 \pm 1.1$ years, including 223 superficial SSI as well as 181 PJIs. The mean age of the patient cohort was $74.2 \pm 22.7$ years, with a mean BMI of $32.3 \pm 6.4 \mathrm{~kg} / \mathrm{m}^{2}$. Patient demographics and surgical variables for the TKA patient cohort are summarized in - Table 1. The causative pathogens for PJIs are summarized in - Table 2. On cross-validation of the training set, the AUCs of the candidate models ranged from 0.78 for SVMs to 0.84 for ANNs ( - Table 3). The calibration intercept ranged from -0.18 to 0.17 , with the best intercept for ANNs (intercept of 0.07; - Table 3). The lowest Brier score error was achieved

Table 3 Discrimination and calibration of machine learning algorithms on the training set for TKA patients

\begin{tabular}{|c|c|c|c|c|c|}
\hline Metric & $\begin{array}{l}\text { Artificial neural } \\
\text { network }\end{array}$ & $\begin{array}{l}\text { Stochastic } \\
\text { gradient boosting }\end{array}$ & $\begin{array}{l}\text { Support vector } \\
\text { machine }\end{array}$ & Random forest & $\begin{array}{l}\text { Elastic-net } \\
\text { penalized } \\
\text { logistic } \\
\text { regression }\end{array}$ \\
\hline \multirow[t]{2}{*}{ AUC } & 0.85 & 0.80 & 0.79 & 0.81 & 0.80 \\
\hline & $(0.82-0.82)$ & $(0.77-0.83)$ & $(0.77-0.81)$ & $(0.79-0.84)$ & $(0.77-0.83)$ \\
\hline \multirow[t]{2}{*}{ Intercept } & 0.07 & 0.16 & -0.18 & -0.12 & 0.17 \\
\hline & $(-0.01$ to 0.15$)$ & $(-0.05$ to 0.37$)$ & $(-0.30$ to -0.06$)$ & $(-0.20$ to -0.04$)$ & $(-0.02$ to 0.36$)$ \\
\hline \multirow[t]{2}{*}{ Slope } & 1.03 & 1.22 & 1.11 & 0.85 & 1.09 \\
\hline & (0.91 to 1.15 ) & (1.07 to 1.37 ) & (1.01 to 1.21 ) & (0.75 to 0.95$)$ & (1.04 to 1.14$)$ \\
\hline \multirow[t]{2}{*}{ Brier } & 0.054 & 0.056 & 0.056 & 0.056 & 0.055 \\
\hline & (0.053 to 0.056$)$ & (0.055 to 0.057$)$ & (0.054 to 0.057$)$ & $(0.055$ to 0.058$)$ & $(0.054$ to 0.056$)$ \\
\hline
\end{tabular}

Abbreviations: AUC, area under the receiver operating characteristic curve; TKA, total knee arthroplasty.

Note: Data was expressed as mean (95\% confidence interval). Null model Brier score $=0.058$. 
Table 4 Discrimination and calibration of machine learning algorithms on the testing set for TKA patients

\begin{tabular}{|l|l|l|l|l|l|}
\hline Metric & $\begin{array}{l}\text { Artificial neural } \\
\text { network }\end{array}$ & $\begin{array}{l}\text { Stochastic gradient } \\
\text { boosting }\end{array}$ & $\begin{array}{l}\text { Support vector } \\
\text { machine }\end{array}$ & $\begin{array}{l}\text { Random } \\
\text { forest }\end{array}$ & $\begin{array}{l}\text { Elastic-net } \\
\text { penalized logistic } \\
\text { regression }\end{array}$ \\
\hline AUC & 0.84 & 0.79 & 0.78 & 0.80 & 0.80 \\
\hline Intercept & 0.09 & 0.18 & -0.21 & -0.17 & 0.18 \\
\hline Slope & 1.06 & 1.27 & 1.15 & 0.90 & 1.10 \\
\hline Brier & 0.054 & 0.055 & 0.056 & 0.055 & 0.054 \\
\hline
\end{tabular}

Abbreviations: AUC, area under the receiver operating characteristic curve; TKA, total knee arthroplasty.

Note: Data was expressed as mean (95\% confidence interval). Null model Brier score $=0.059$.

by ANNs (Brier score of 0.054). In the testing set, the AUCs of the five candidate models ranged from 0.78 to 0.84 ( - Table 4). The highest AUC was achieved by ANNs (AUC $=0.84$; - Table 4). The Brier score errors in the testing set varied between 0.054 and 0.056 , with the lowest Brier score error for ANNs. The accuracy of the five ML models exceeded $94 \%$.

Decision curve analysis showed a higher net benefit for all five ML models, when compared with the default strategies of changing management for all patients or no patients. Variables significantly associated with the development of SSI were old age ( $>75$ years), male gender, CCI, smoking, alcohol use, diabetes, Medicare insurance, and BMI ( - Fig. $\mathbf{1}$ ). The strongest predictors of SSI were CCI, BMI $\left(>30 \mathrm{~kg} / \mathrm{m}^{2}\right)$, and smoking (-Fig. 2). Numerous medical comorbidities demonstrated only a small impact on the development of SSI following primary TKA: drug abuse (6.4\%), depression (5.3\%), renal failure (5.1\%), malignant tumor (3.8\%), and hypertension (1.2\%). In terms of model performance, there was no significant difference for ML candidate models predictions between superficial SSI and PJIs considering the AUC $(p=0.35)$, calibration intercept $(p=0.47)$, calibration slope $(p=0.51)$, and Brier score $(p=0.44$; - Fig. 3$)$.

An example of a local, individual patient-level explanation for the model predictions by ANN is shown in - Fig. 4. For a 63-year old obese (BMI: $41 \mathrm{~kg} / \mathrm{m}^{2}$ ) TKA patient with CCI of 3.03, diabetes, and Medicare insurance, who had no history of alcohol and smoking, the predicted probability of SSI following primary TKA was $16.3 \%$. A high CCI, high BMI (> $35 \mathrm{~m} / \mathrm{kg}^{2}$ ), diabetes, and Medicaid insurance status increased the probability of SSI, whereas age, no prior history of alcohol use, and smoking decreased the probability of SSI.

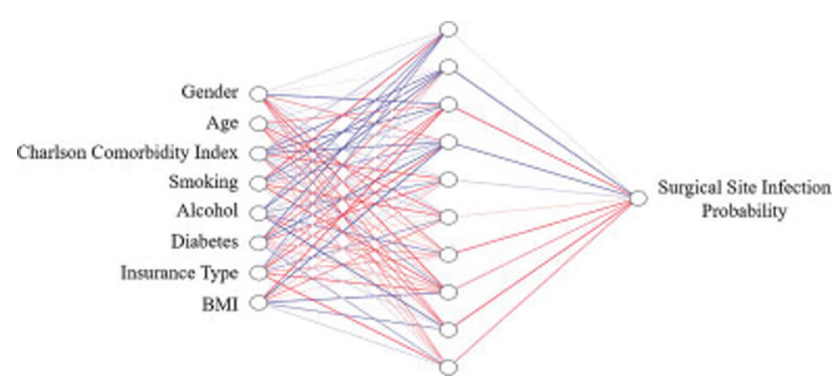

Fig. 1 Machine learning model for the prediction of surgical site infection following primary total knee arthroplasty.

\section{Discussion}

Due to the growing attention to predict SSIs following TKA and to optimize risk factors preoperatively, ${ }^{12}$ there is an increasing interest in applying ML models to TKA patient care with regard to postsurgical infections. In this present study, we demonstrate excellent performance for all five ML candidate models on discrimination, calibration, and decision curve analysis in terms of predicting superficial and deep SSIs in patients following primary TKA, with the ANN demonstrating the strongest performance of all ML models (AUC $=0.84)$. Prior works aiming to predict SSI following hip and knee TJA did not achieve excellent model performance. A retrospective database study by Inacio et $\mathrm{al}^{13}$ used a prescription-based comorbidity measure to predict PJI within 90 days following hip and knee TJA, reporting an AUC of less than 0.63 for their modeling techniques. Similarly, Shah et al did not achieve excellent model predictions (AUC $=0.73$ ) in their ML study, which intended to predict postoperative complications such as SSI in patients following primary hip and knee TJA. ${ }^{14}$ Our current ML models additionally show a higher AUC, when compared with recent ML models for the prediction of SSIs following spinal fusion $(A U C=0.77)$ and neurological operations (AUC $=0.76$ ). ${ }^{9,15}$ Based on the high accuracy of our ANN models, when compared with prior literature, the presented ANN models have the potential to be used in real-time patient-specific SSI prediction in primary TKA patients.

Based on the results of the presented ML models, the strongest predictors of superficial and deep SSIs following primary TKA were $\mathrm{CCI}$, obesity $\left(\mathrm{BMI}>30 \mathrm{~kg} / \mathrm{m}^{2}\right.$ ), and smoking. The model performance did not significantly differ between predictions for superficial and deep SSIs, probably due to the fact that prior systematic reviews showed strong concurrence in terms of risk factors for superficial and deep SSIs. ${ }^{16}$ The CCI, the most influential predictor of SSIs, initially designed to predict mortality, is a highly validated mechanism for quantifying patient comorbidities. ${ }^{17}$ More recently, the $\mathrm{CCI}$ has been studied as a predictive tool for various events following primary TJA, including complications, ${ }^{18}$ readmission rates, ${ }^{19}$ functional outcomes, ${ }^{20}$ discharge disposition, ${ }^{21}$ and prolonged length of stay. ${ }^{19}$ Additionally, the $\mathrm{CCI}$ has been shown to be the predictor of greater hospital charges and costs associated with TKA. ${ }^{22}$ Furthermore, 


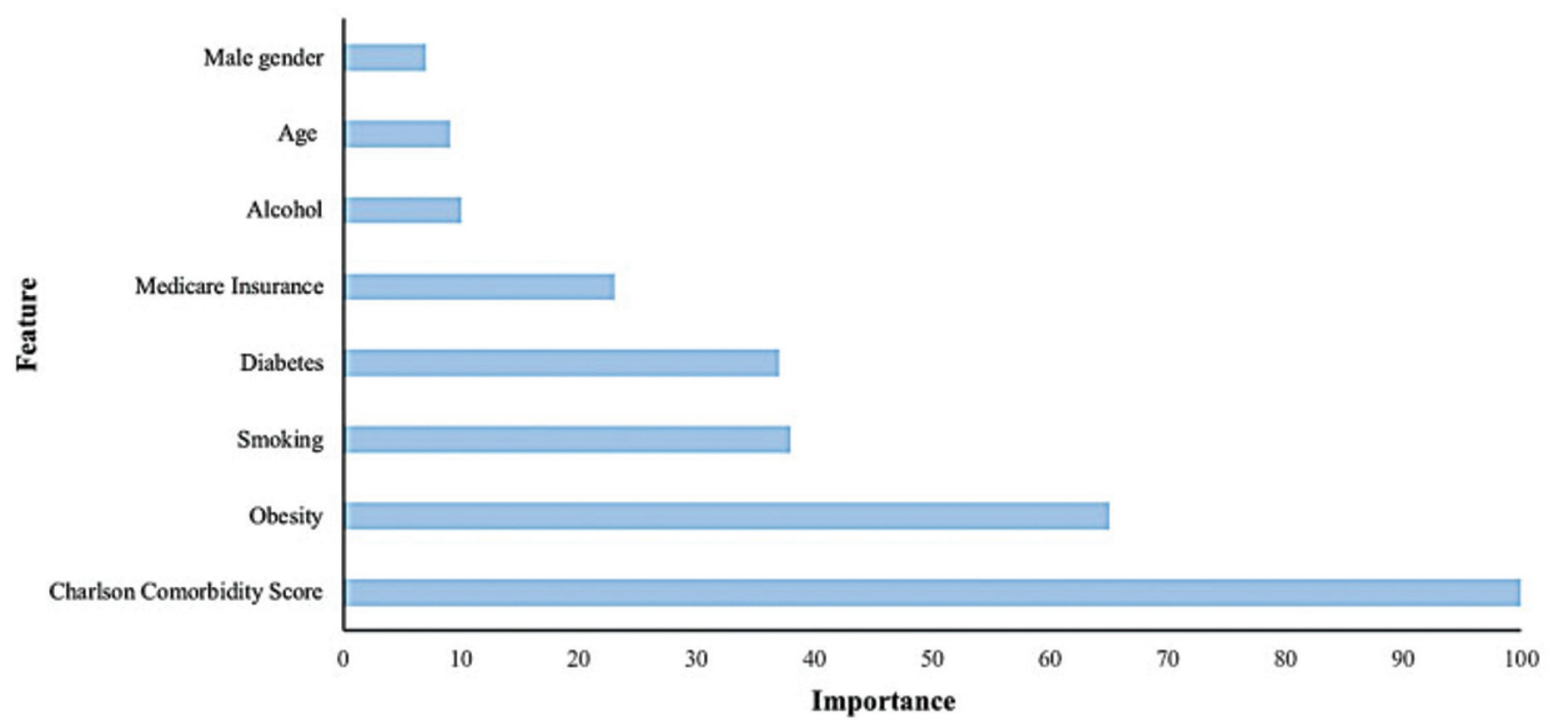

Fig. 2 Global variable importance plot for the prediction of surgical site infection following primary TKA. TKA, total knee arthroplasty.

several previous studies support our observation that a higher number of comorbidities, identified through the $\mathrm{CCI}$, were associated with a higher risk of SSI. ${ }^{7,13}$

From this present ANN model, obesity (BMI $>30 \mathrm{~kg} / \mathrm{m}^{2}$ defined as per recommendations of the US Center for Disease Control $^{23}$ ) was the second strongest predictor of superficial and deep SSIs following primary TKA. Several meta-analyses have analyzed the relationship between SSI and obesity. ${ }^{24}$ Although there is no clear cutoff value, a higher BMI is known to be associated with an increased risk of SSI following TKA, indicating that BMIs greater than $40 \mathrm{~kg} / \mathrm{m}^{2}$ are strongly correlated with SSIs. ${ }^{25}$ For this, a plausible explanation may lie in the observations of previous literature which demonstrated that obese TKA patients had increased blood loss, longer surgical times, increased comorbid conditions, and prolonged postoperative wound drainage. ${ }^{26,27}$ In terms

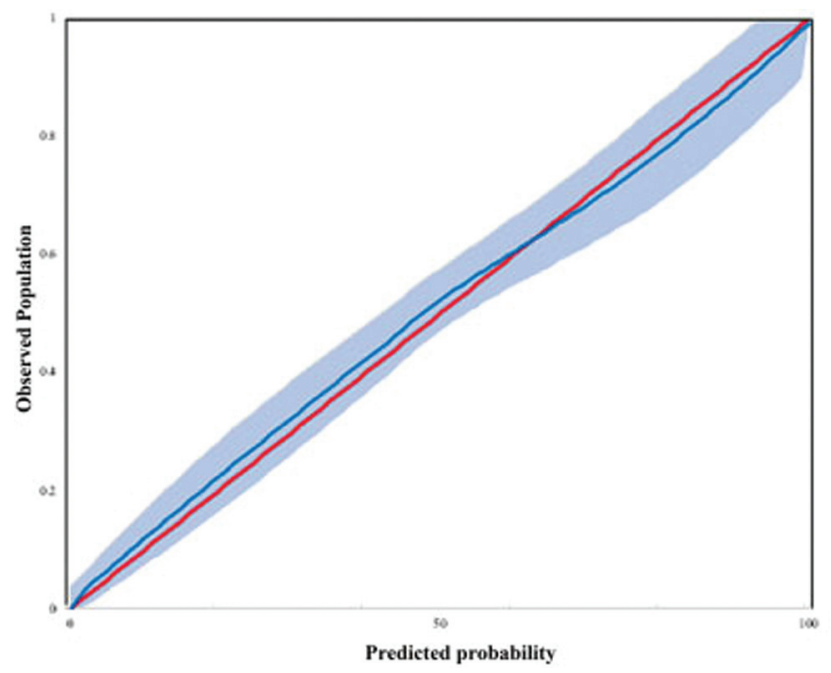

Fig. 3 Calibration plot for the neural network model for the prediction of surgical site infection following primary TKA. TKA, total knee arthroplasty. of smoking as a strong predictor of SSI following index TKA, similar to the findings of this present study, a large database study by Kremers et al has provided comparable outcomes with an increased risk of SSI following primary THA and TKA in smokers. ${ }^{28}$ Several prior works reported that approximately $7 \%$ of TKA patients were current tobacco users, ${ }^{29}$ and smoking placed patients at a 3.5\% increased risk of SSI following TKA, when compared with non-smoking TKA patients. ${ }^{30}$ Furthermore, the most common cause of revision TKA in smokers is PJI. ${ }^{31}$

There is a strong agreement in terms of risk factors of SSI development following primary TKA between the present ML study and prior retrospective work. ${ }^{26,28}$ However, the present study identified increased importance of obesity than previously reported. ${ }^{8,32}$ With previous studies reporting a risk of $1-2 \%$ for the development of PJI solely due to obesity, ${ }^{32}$ this present ML study shows a greater significance of a high BMI (3.8\%). This may be based on the increased accuracy of data analysis as provided by ML algorithms,

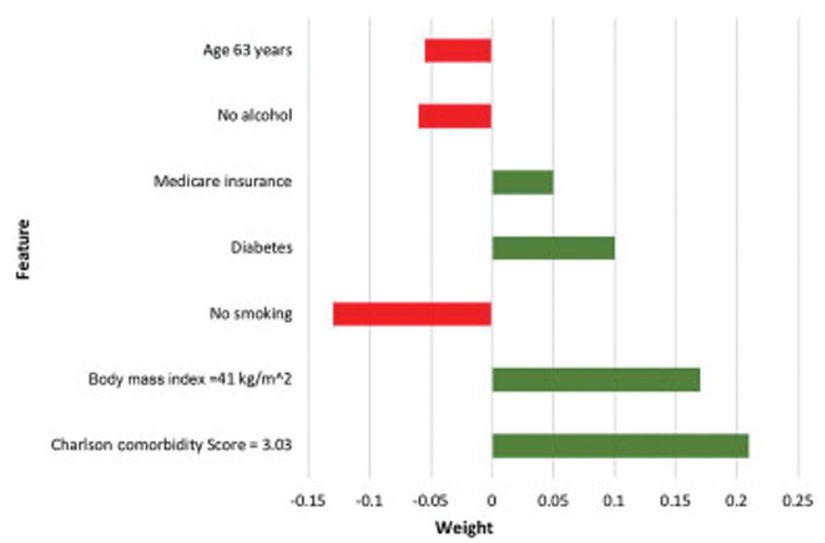

Fig. 4 Example of individual patient-specific explanation generated by the neural network model for TKA patients. TKA, total knee arthroplasty. 
which possess the ability to accurately identify complex relationships between clinical variables, even in noisy and incomplete datasets. ${ }^{33}$ Furthermore, ML algorithms provide risk factor estimates within seconds, thereby providing a viable tool to assist clinical decision-making. Consequently, ML tools have seen a rapid rise over recent years in many fields of medicine. The use of ML algorithms in clinical environments has the potential to support clinical decision-making through a data-driven driven approach that provides highly accurate results in real time. Therefore, it can overcome prior approaches that solely rely on the experience of orthopaedic surgeons, which may be of benefit especially in patients with complex medical history. However, there may be concerns associated with the use of ML technology regarding the limited access to health care institutions for patients at the risk of postoperative complications. This is based on the current reimbursement models, where patients with an increased amount of postoperative complications are less profitable to the health care institutions; therefore, these patients may face challenges to access health care providers.

The results of the current study provide practical information that may be clinically useful for the preoperative identification of patients with a high probability of superficial or deep SSI following primary TKA, solely predicted by ML models from patient demographic data and medical comorbidities. To be used as a reliable predictive tool in clinical practice, the ability to accurately classify the good and the poor prognosis is required. Since the occurrence of SSI is strongly associated with poor outcomes following primary TKA, this stratification using ANN models could be helpful to arthroplasty surgeons and patients during preoperative counseling and patient optimization based on preoperative patient data. Additionally, for high-risk TKA patients, determined through their estimated risk for SSI, an extra-preventative health care resource could be applied to optimize modifiable risk factors to minimize the risk of SSI. These extra-preventative health care resources may include preoperative rehabilitation programs as well as preoperative counseling and educational seminars to optimize TKA patients prior to surgery.

The present study has several potential limitations. First, this study has inherent disadvantages of a retrospective study design such as bias and an inability to control confounding factors. Second, although a large number of primary TKA patients were included in this single-institution study, the number of patients with postoperative SSI was still relatively small. Selection bias from a limited database, an inseparable limitation of ML models, can render poor prognostic generalizability of the presented ML models. To address this selection bias, larger datasets sampling patients with a broad demographic spectrum may be needed in future studies. Nonetheless, the presented five ML algorithms demonstrated an accuracy of greater than $94 \%$, highlighting the strong predictive ability of these computational tools. Third, the ML models were only validated internally, which may limit the generalizability in clinical practice as there may be differences in the patient population between our tertiary referral center and alternative health care providers across the country.
Finally, most of the patient risk factors evaluated in this study were listed as binary including the presence of depression or diabetes or alcohol consumption; thus, the effect of disease severity was not evaluated in this study. However, similar limitations were reported in prior retrospective studies on this topic. $^{34-36}$

In conclusion, this study developed and validated five ML models for the prediction of patient-specific SSI following primary TKA. The study findings show excellent model performance, with the best modeling accuracy of ANNs. This highlights the potential of these computational models to assist in preoperative patient optimization and counseling to maximize outcomes in TKA patients.

Conflict of Interest

None declared.

\section{References}

1 Sloan M, Premkumar A, Sheth NP. Projected volume of primary total joint arthroplasty in the U.S., 2014 to 2030. J Bone Joint Surg Am 2018;100(17):1455-1460

2 Edwards JR, Peterson KD, Mu Y, et al. National Healthcare Safety Network (NHSN) report: data summary for 2006 through 2008, issued December 2009. Am J Infect Control 2009;37(10):783-805

3 National Nosocomial Infections Surveillance System. National Nosocomial Infections Surveillance (NNIS) System Report, data summary from January 1992 through June 2004, issued October 2004. Am J Infect Control 2004;32(08):470-485

4 Kurtz S, Ong K, Lau E, Mowat F, Halpern M. Projections of primary and revision hip and knee arthroplasty in the United States from 2005 to 2030. J Bone Joint Surg Am 2007;89(04):780-785

5 Shohat N, Bauer T, Buttaro M, et al. Hip and knee section, what is the definition of a periprosthetic joint infection (PJI) of the knee and the hip? Can the same criteria be used for both joints?: Proceedings of international consensus on orthopedic infections. J Arthroplasty 2019;34(2S):S325-S327

6 Berríos-Torres SI, Umscheid CA, Bratzler DW, et al; Healthcare Infection Control Practices Advisory Committee. Centers for disease control and prevention guideline for the prevention of surgical site infection, 2017. JAMA Surg 2017;152(08):784-791

7 Bozic KJ, Lau E, Kurtz S, et al. Patient-related risk factors for periprosthetic joint infection and postoperative mortality following total hip arthroplasty in Medicare patients. J Bone Joint Surg Am 2012;94(09):794-800

8 Klemt C, Tirumala V, Smith EJ, Padmanabha A, Kwon Y-M. Development of a preoperative risk calculator for re-infection following revision surgery for periprosthetic joint infection. J Arthroplasty 2021;36(02):693-699

9 Hopkins BS, Mazmudar A, Driscoll C, et al. Using artificial intelligence (AI) to predict postoperative surgical site infection: a retrospective cohort of 4046 posterior spinal fusions. Clin Neurol Neurosurg 2020;192(January):105718

10 Parvizi J, Zmistowski B, Berbari EF, et al. New definition for periprosthetic joint infection: from the Workgroup of the Musculoskeletal Infection Society. Clin Orthop Relat Res 2011;469 (11):2992-2994

11 Ferro CAT. Comparing probabilistic forecasting systems with the Brier score. Weather Forecast 2007;22(05):1076-1088

12 Bozic KJ, Ong K, Lau E, et al. Estimating risk in Medicare patients with THA: an electronic risk calculator for periprosthetic joint infection and mortality. Clin Orthop Relat Res 2013;471(02): 574-583

13 Inacio MCS, Pratt NL, Roughead EE, Graves SE. Predicting infections after total joint arthroplasty using a prescription based comorbidity measure. J Arthroplasty 2015;30(10):1692-1698 
14 Shah AA, Devana SK, Lee C, Kianian R, van der Schaar M, SooHoo NF. Development of a novel, potentially universal machine learning algorithm for prediction of complications after total hip arthroplasty. J Arthroplasty 2021;36(05):1655-1662

15 Tunthanathip T, Sae-Heng S, Oearsakul T, Sakarunchai I, Kaewborisutsakul A, Taweesomboonyat C. Machine learning applications for the prediction of surgical site infection in neurological operations. Neurosurg Focus 2019;47(02):E7

16 Antonelli B, Chen AF. Reducing the risk of infection after total joint arthroplasty: preoperative optimization. Arthroplasty 2019;1 (04):1-13

17 Werner BC, Higgins MD, Pehlivan HC, Carothers JT, Browne JA. Super obesity is an independent risk factor for complications after primary total hip arthroplasty. J Arthroplasty 2017;32(02):402-406

18 Singh JA, Jensen MR, Harmsen WS, Gabriel SE, Lewallen DG. Cardiac and thromboembolic complications and mortality in patients undergoing total hip and total knee arthroplasty. Ann Rheum Dis 2011;70(12):2082-2088

19 Rozell JC, Courtney PM, Dattilo JR, Wu CH, Lee GC. Should all patients be included in alternative payment models for primary total hip arthroplasty and total knee arthroplasty? J Arthroplasty 2016;31(9 Suppl)45-49

20 Johnsen SP, Sørensen HT, Pedersen AB, Lucht U, Søballe K, Overgaard S. Patient-related predictors of implant failure after primary total hip replacement in the initial, short- and long-term: a nationwide Danish follow-up study including 36984 patients. J Bone Jt Surg - Ser B 2006;88(10):1303-1308

21 Schwarzkopf R, Ho J, Snir N, Mukamel DD. Factors influencing discharge destination after total hip arthroplasty: a California State Database Analysis. Geriatr Orthop Surg Rehabil 2015;6(03):215-219

22 Shah AN, Vail TP, Taylor D, Pietrobon R. Comorbid illness affects hospital costs related to hip arthroplasty: quantification of health status and implications for fair reimbursement and surgeon comparisons. J Arthroplasty 2004;19(06):700-705

23 Caballero B. Humans against obesity: who will win? Adv Nutr 2019;10(Suppl 1):S4-S9

24 Kunutsor SK, Whitehouse MR, Blom AW, Beswick ADINFORM Team. Patient-related risk factors for periprosthetic joint infection after total joint arthroplasty: a systematic review and metaanalysis. PLoS One 2016;11(03):e0150866

25 Alvi HM, Mednick RE, Krishnan V, Kwasny MJ, Beal MD, Manning DW. The effect of BMI on 30 day outcomes following total joint arthroplasty. J Arthroplasty 2015;30(07):1113-1117
26 Duchman KR, Pugely AJ, Martin CT, Gao Y, Bedard NA, Callaghan JJ. Operative time affects short-term complications in total joint arthroplasty. J Arthroplasty 2017;32(04):1285-1291

27 Patel VP, Walsh M, Sehgal B, Preston C, DeWal H, Di Cesare PE. Factors associated with prolonged wound drainage after primary total hip and knee arthroplasty. J Bone Joint Surg Am 2007;89 (01):33-38

28 Maradit Kremers H, Kremers WK, Berry DJ, Lewallen DG. Social and behavioral factors in total knee and hip arthroplasty. J Arthroplasty 2015;30(10):1852-1854

29 Singh JA, Schleck C, Harmsen WS, Jacob AK, Warner DO, Lewallen DG. Current tobacco use is associated with higher rates of implant revision and deep infection after total hip or knee arthroplasty: a prospective cohort study. BMC Med 2015;13:283

30 Springer BD. Modifying risk factors for total joint arthroplasty: strategies that work nicotine. J Arthroplasty 2016;31(08): $1628-1630$

31 Kapadia BH, Issa K, Pivec R, Bonutti PM, Mont MA. Tobacco use may be associated with increased revision and complication rates following total hip arthroplasty. J Arthroplasty 2014;29(04): 777-780

32 Tan TL, Maltenfort MG, Chen AF, et al. Development and evaluation of a preoperative risk calculator for periprosthetic joint infection following total joint arthroplasty. J Bone Joint Surg Am 2018;100(09):777-785

33 Bini SA. Artificial intelligence, machine learning, deep learning, and cognitive computing: what do these terms mean and how will they impact health care? J Arthroplasty 2018;33(08):2358-2361

34 Klemt C, Smith EJ, Tirumala V, Bounajem G, van den Kieboom J, Kwon Y-M. Outcomes and risk factors associated with 2-stage reimplantation requiring an interim spacer exchange for periprosthetic joint infection. J Arthroplasty 2021;36(03):1094-1100

35 Klemt C, Tirumala V, Oganesyan R, Xiong L, van den Kieboom J, Kwon Y-M. Single-stage revision of the infected total knee arthroplasty is associated with improved functional outcomes: a propensity score-matched cohort study. J Arthroplasty 2021;36 (01):298-304

36 Shohat N, Goswami K, Tan TL, et al; ESCMID Study Group of Implant Associated Infections (ESGIAI) and the Northern Infection Network of Joint Arthroplasty (NINJA) 2020 Frank Stinchfield Award: identifying who will fail following irrigation and debridement for prosthetic joint infection. Bone Joint J 2020;102-B (7_Supple_B):11-19 